\title{
Editorial \\ Gleichstellung der Geschlechter im Bildungsbereich
}

\section{Lucien Criblez, Karin Gottschall und Danièle Périsset}

Die «Schweizerische Zeitschrift für Bildungswissenschaften» hat letztmals im Jahr 2000 eine Themennummer zu Genderfragen publiziert, damals speziell auf Genderfragen in der beruflichen Bildung ausgerichtet. Inzwischen haben weitere Massnahmen die Gleichstellung befördert und für den Bildungsbereich lässt sich eine weitgehende formale Gleichstellung feststellen: Mädchen und Knaben, junge Frauen und junge Männer sowie Erwachsene beiden Geschlechts haben im Hinblick auf die Nutzung von Bildungsmöglichkeiten formal die gleichen Chancen und Möglichkeiten.

Trotzdem nutzen Frauen und Männer diese Möglichkeiten bis heute in sehr unterschiedlicher Art und Weise, Frauen sind in vielen Bereichen sowohl des allgemeinbildenden Teils des Bildungswesens als auch des berufsbildenden Teils anders vertreten als Männer. Auch heute noch haben Berufe sehr eindeutig ein Geschlecht und das Bildungswesen der Sekundarstufe II und des tertiären Bildungsbereichs ist nach wie vor stark von geschlechtsspezifischen Berufs- und Laufbahnwahlen geprägt. Auch der Lehrberuf ist stark genderspezifisch segregiert (BfS, 2013a): Während im Kindergarten und der Primarschule vor allem Frauen unterrichten, sind es im höheren Bildungswesen nach wie vor mehrheitlich Männer. Gleichzeitig zeigen sich an einigen Stellen des Bildungswesens neue Phänomene, deren Zustandekommen noch nicht wirklich erklärt ist und deren Konsequenzen sich erst allmählich abzeichnen: Die gymnasiale Maturitätsquote liegt bei jungen Frauen seit 1993 höher als bei jungen Männern (BfS, 2013b) und inzwischen studierenden mehr junge Frauen als junge Männer an Universitäten (BfS, 2013c). Trotzdem wirkt die sogenannte «leaky pipeline» nach wie vor: Wenn es um wissenschaftliche Laufbahnen (vgl. Leemann \& Stutz, 2010; Schubert \& Engelage, 2011) oder Spitzenpositionen in Gesellschaft, Wirtschaft (Rigassi \& Büsser, 2014) und Politik ${ }^{1}$ geht, sind die Frauen nach wie vor untervertreten.

Um solche und ähnliche Fragen in andern gesellschaftlichen Bereichen zu analysieren hat der Bundesrat 2007 den Schweizerischen Nationalfonds zur Förderung der wissenschaftlichen Forschung (SNF) mit der Durchführung 
eines Nationalen Forschungsprogramms zur Gleichstellung der Geschlechter (NFP 60) beauftragt ${ }^{2}$. Im Mai 2009 wurde ein zweiphasiges Ausschreibungsverfahren auf der Grundlage des Ausführungsplans eröffnet. 2010 und 2011 haben die 21 positiv evaluierten Projekte ${ }^{3}$ ihre Arbeit aufgenommen, das Programm wurde mit mehreren Veranstaltungen 2014 abgeschlossen (vgl. Leitungsgruppe NFP 60, 2014). Die Projekte waren thematisch drei grösseren Clustern zugeordnet: Arbeit + Organisation, Bildung + Karriere sowie Familie + Privathaushalt. Das NFP 60 zielte darauf, neues Wissen und neue Erkenntnisse in diesen drei "Clustern» zu generieren, indem die Politikprozesse im Bereich der Gleichstellungspolitik rekonstruiert, Gleichstellungsmassnahmen evaluiert und verschiedene problemorientierte Analysen zu den Themenbereichen Familie, Erwerbsarbeit und Bildung vorgenommen werden.

Die vorliegende Themennummer präsentiert einerseits Resultate zum Thema «Gleichstellung der Geschlechter im Bildungsbereich» aus einigen im Rahmen des NFP 60 durchgeführten Projekten, andererseits weitere Forschungsresultate zum Thema. Die Beiträge fokussieren primär auf den geografischen Raum Schweiz oder thematisieren Gleichstellungsaspekte im Bildungsbereich, die für die Entwicklung in der Schweiz von Bedeutung sind. Ein besonderes Interesse galt drei Bereichen: der familienergänzenden Kinderbetreuung, den geschlechtstypischen und -untypischen Ausbildungs-, Berufs- und Laufbahnwahlen sowie der geschlechtsspezifischen Segmentierung des tertiären Bildungsbereichs.

Die Beiträge sind in der Themennummer - soweit dies überhaupt möglich und sinnvoll ist - entlang einer Lebenslaufperspektive geordnet. Dieses Ordnungsprinzip ist nicht nur der Systematik des Bildungssystem geschuldet: Die vielfältigen Ergebnisse aus dem NFP 60 weisen sehr deutlich auf kumulative Effekte mangelnder Gleichstellung über die Lebensphasen hin.

Der Beitrag von Franziska Vogt, Julia Nentwich und Wiebke Tennhoff untersucht die Interaktionen im Krippenalltag. Die Analyse von Videodaten zielt darauf ab, in den Interaktionen Situationen von doing und undoing gender zu identifizieren und sie daraufhin zu prüfen, ob die Kinderbetreuenden in solchen Situationen mit Dramatisierung oder Dethematisierung reagieren. In den Fallstudien (vier Kinderkrippen) konnten deutliche Unterschiede in beiderlei Hinsicht festgestellt werden. Die Autorinnen führen diese Unterschiede einerseits auf unterschiedliche pädagogische Überzeugungen, andererseits aber vor allem auch auf die Organisationskultur in Kinderkrippen zurück. Sie sehen Veränderungspotenzial für Leitungspersonen und Teams deshalb vor allem im Bereich der Organisationsentwicklung.

Wiebke Bobeth-Neumann stellt in ihrem Beitrag einen Erklärungsansatz für den empirisch evidenten Tatbestand der disproportionalen Geschlechterverhältnisse bei Schulleitenden vor. Auf der Grundlage von Interviews mit deutschen Grundschullehrkräften und vor dem Hintergrund der Bourdieu'schen Feldtheorie und seiner Unterscheidung zwischen sozialem, kulturellem und ökono- 
mischen Kapital sieht die Autorin den Hauptgrund für die unterproportionale Vertretung der Schulleiterinnen in unterschiedlichen Rollenerwartungen des sozialen Feldes. Während die "Karriere» von Grundschullehrerinnen zu Schulleiterinnen als «verbissener» Aufstieg interpretiert wird und Ermutigungen für einen solchen Aufstieg aus dem sozialen Umfeld weitgehend ausbleiben, gilt der entsprechende Karriereschritt bei Grundschullehrern als «natürlich» und zielorientiert und wird frühzeitig unterstützt.

Im Beitrag von Farinaz Fassa und Céline Naef werden zwei Gleichstellungsmassnahmen der Gleichstellungsbüros auf der Grundlage von Interviews mit Akteurinnen und Akteuren im entsprechenden Feld untersucht: einerseits der Zukunftstag (früher: Mädchentag), der zuerst die Mädchen, heute alle Jugendlichen für eine geschlechterunabhängige Berufswahl sensibilisieren soll, andererseits ein Lehrmittelpaket mit vier Broschüren der Westschweizer Erziehungsdirektorenkonferenz, das Lehrpersonen im Hinblick auf einen gleichstellungsorientierten Unterricht unterstützen soll: «L'école de l'égalité». Die unterschiedliche Akzeptanz der beiden Massnahmen führen die Autorinnen darauf zurück, dass beim Mädchen- bzw. Zukunftstag nicht so sehr die Gleichstellung der Geschlechter im Vordergrund stünde, sondern die berufliche Orientierung - und damit eine zentrale wirtschaftliche Frage. Das Lehrmittelpaket jedoch stelle die traditionellen Geschlechterhierarchien, auch in Familie und Haushalt, in Frage und habe dazu geführt, dass die Schulleitungen kaum aktiv geworden seien, um die Implementation zu unterstützen.

Belinda Aeschlimann, Walter Herzog und Elena Makarova haben die Frage untersucht, weshalb Gymnasiastinnen und Gymnasiasten ein Studium im MINT-Bereich (Mathematik, Informatik, Naturwissenschaft und Technik) wählen bzw. abwählen. Insgesamt zeigen die Resultate, dass schon die generelle Bereitschaft, ein MINT-Fach für das Studium zu wählen, eher gering ist und dass insbesondere junge Frauen geringes Interesse am MINT-Bereich zeigen. Allerdings zeigte sich, dass neben dem Geschlecht zugleich andere Faktoren von Bedeutung sind, die den Geschlechtereffekt teilweise neutralisieren. Bei Gymnasiastinnen begünstigt die Herkunft aus sozioökonomisch besser gestellten Familien die Wahrscheinlichkeit, ein MINT-Studium zu wählen. Soziale Orientierung und intrinsische Motivation sind bei Gymnasiastinnen stärker ausgeprägt als Gymnasiasten - begünstigen aber die Abwahl von MINT-Fächern. Auch schulische Faktoren beeinflussen die Fächerwahl: Gute Leistungen im MINT-Bereich beeinflussen die entsprechende Studienwahl positiv, aber junge Frauen zeigen bei gleich guter Leistung bedeutend weniger Interesse am MINT-Bereich als junge Männer.

Wie schätzen Gymnasiastinnen und Gymnasiasten die Nützlichkeit von Mathematik ein, wie schätzen sie ihre Leistungen ein, wie sind ihre Kontrollüberzeugungen, ihre positiven und negativen Emotionen gegenüber dem Fach? Wie kontrollieren sie diese Emotionen, wie viel investieren sie in dieses Fach und schätzen sie das Fach als «männliches» Fach ein? Diese Fragen analysieren 
Philippe Genoud, Gabriel Kappeler und Matthias Guillod auf der Grundlage einer Fragebogenerhebung bei etwas mehr als 400 Gymnasiastinnen und Gymnasiasten. Neben Resultaten, die bekannte Ergebnisse aus andern Studien auch fürs Gymnasium bestätigen, haben die Autoren auch Profile von Schülerinnen und Schülern identifiziert: So können sie zeigen, dass sowohl leistungsstarke männliche Jugendliche als auch leistungsschwache weibliche Jugendliche über geschlechterstereotype Vorstellungen über den Mathematikunterricht verfügen. Daneben identifizieren sie unter anderem ein Profil ängstlicher Schülerinnen und Schüler, die mittlere Leistungen erbringen, vom Nutzen des Faches überzeugt sind und auch entsprechend ins Fach investieren, die sich aber vom Stress überwältigen lassen und kaum über Stressbewältigungsstrategien verfügen.

Simone Berweger, Christa Kappeler, Andrea Keck Frei und Christine Bieri Buschor fokussieren in ihrem Beitrag das Interesse von männlichen Gymnasiasten für den Lehrerberuf und identifizieren auf der Grundlage einer Fragebogenerhebung bei mehr als 1000 Zürcher Gymnasiastinnen und Gymnasiasten Faktoren, welche dieses Interesse beeinflussen. Rund die Hälfte der Gymnasiasten verfügte zum Untersuchungszeitpunkt (16-Jährige) noch über keine Studienoder Berufspräferenzen. Diejenigen, die sich bereits entschieden haben, sehen eher geschlechtsspezifische Laufbahnwahlen vor, das Interesse am Lehrberuf ist bei jungen Männern im Vergleich zu jungen Frauen sehr gering. Gymnasiasten halten die Laufbahnoption «Lehrer» im Vergleich zu andern Optionen für unattraktiv. Positiv werden sie durch ein väterliches Rollenmodell beeinflusst, aber auch die Möglichkeit, den Beruf mit einer zeitintensiven Zusatzbeschäftigung (Sport) vereinbaren zu können, spielt eine bestimmte Rolle. Entscheiden sie sich für den Lehrberuf, ist das Interesse am Beruf der bedeutendste Faktor. Kein Effekt zeigt bei Gymnasiasten die Möglichkeit der Vereinbarkeit von Familie und Beruf.

Auf der Grundlage von Daten aus der Zürcher Längsschnittstudie «Von der Schulzeit bis zum mittleren Erwachsenenalter» gehen Kurt Häfeli, Achim Hättich, Claudia Schellenberg und Nicolas Schmaeh den Gründen für persistierende Geschlechterunterschiede beim Berufserfolg nach. Die Resultate zeigen erstens deutliche Einflüsse der sozialen Herkunft und der Persönlichkeitsmerkmale, aber auch solche des Ausbildungsniveaus oder des Berufsstatus mit 19 Jahren. Die horizontale und vertikale Mobilität ist bei Frauen deutlich geringer als bei Männern. Bei der Generation der heute 50-Jährigen zeigt sich eine gute berufliche Etablierung durch Berufswechsel und Aufstieg bei den Männern, vor allem zwischen 20 und 35 Jahren, eine deutliche Festlegung der beruflichen Laufbahn bereits in jungen Jahren bei den Frauen. Spätere Karriereschritte werden bei Frauen durch die Familienphase, Teilzeitarbeit und die traditionelle Rollenteilung in der Familienarbeit nur bedingt möglich.

Die hier versammelten Beiträge liefern wichtige Erkenntnisse zu strukturellen und kulturellen, institutionellen wie individuellen Bedingungen, die eine Umsetzung formaler Gleichstellung restringieren oder begünstigen und 
verweisen zugleich auf weiteren Forschungs- und politischen Handlungsbedarf in diesem Feld.

\section{Anmerkungen}

1 Vgl. http://www.bfs.admin.ch/bfs/portal/de/index/themen/17/02/blank/key/frauen_und _politik/kantone.html; Zugriff am 10.6.2015.

2 Vgl. http://www.nfp60.ch/D/Seiten/home.aspx; Zugriff am 10.06.2015.

3 Vgl. http://www.nfp60.ch/D/projekte/Seiten/default.aspx; Zugriff am 10.06.2015.

\section{Literatur}

BfS [Bundesamt für Statistik] (2013a). Die Ausbildung der künftigen Lehrkräfte in der Schweiz. Studierende und Abschlüsse 2012. Neuchâtel: BfS.

BfS (2013b). Maturitäten und Übertritte an Hochschulen 2012. Neuchâtel: BfS.

Leemann, R. J. \& Stutz, H. (Hrsg.) (2010). Forschungsförderung aus Geschlechterperspektive Zugang, Bedeutung und Wirkung in wissenschaftlichen Laufbahnen. Chur, Glarus: Rüegger. Leitungsgruppe NFP 60 (2014). NFP 60 - Gleichstellung der Geschlechter. Ergebnisse und Impulse. Synthesebericht. Bern: SNF. Zugriff am 10.06.2015 unter http://www.nfp60.ch/ SiteCollectionDocuments/NFP_60_Synthese_de_Web.pdf

Schubert, F. \& Engelage, S. (2011). Wie undicht ist die Pipeline? Wissenschaftskarrieren von Frauen in der Schweiz. Kölner Zeitschrift für Soziologie und Sozialpsychologie, 63(3), 431-457. 
